\title{
Numerical simulation investigations of the dynamic control process and frequency response characteristics in an adaptive optics system
}

\author{
Hai-Xing Yan*, Shu-Shan Li, She Chen \\ (Institute of Mechanics, Chinese Academy of Sciences, Beijing 100080, China)
}

\begin{abstract}
The dynamic control process in an adaptive optics (AO) system is the third important factor to affect the performances of the AO system. A numerical simulation of the dynamic control process in an AO system is carried out for the first time to complete a comprehensive numerical simulation of an $\mathrm{AO}$ system. By means of the numerical simulation the long-exposure Strehl ratio and other useful metrics for evaluating the performances of an AO system can be obtained. In comparison to the theoretical analyses of automatic control theory, a numerical simulation of the dynamic control process in an AO system is advantageous. The frequency response characteristics are closely related to the dynamic control performance of an AO system. The bandwidth, phase margin and gain margin which are very important parameters for experimenters can be obtained conveniently from a numerical simulation of the frequency response characteristics. A numerical simulation of the frequency response characteristics in an AO system is presented in this paper for the first time. The numerical simulation results agree with the experimental data excellently. The simulation computational results of frequency response by using composite input of multiple frequencies compare to the single-frequency simulation results very well and can be used to determine the practical parameters of bandwidth and margins. The performances of an AO system can be effectively investigated by means of a combination of a numerical simulation of frequency response characteristics with that of the dynamic control process.
\end{abstract}

Keywords: adaptive optics system, numerical simulation, dynamic control process, frequency response, PI (Proportional plus Integral) controller, bandwidth, phase margin, gain margin

\section{INTRODUCTION}

Adaptive optics (AO) technology has been widely used to improve the image quality in the astronomical observation of ground-based telescope and the laser beam propagation in the turbulent atmosphere. ${ }^{1,2}$ An AO system itself is a complex combination of electro-optic engineering and automatic control technology. In such a complex system modeling and numerical simulation should be very valuable from design, manufacturing, system integration, examination, to practical application.

In the past decade we have been pursuing modeling and numerical simulation in an AO system. Modeling and numerical simulation of laser propagation in the turbulent atmosphere and an AO system in a stationary state were carried out. ${ }^{3}$ In a companion paper $^{4}$, modeling and numerical simulation of the effects of noise and detection error on an AO system are

* Correspondence: Email: hxyan@imech.ac.cn; Phone: (86) 10 62554123; Fax: (86) 1062561284 
reported. The dynamic control process is the third important factor to affect the performances of an AO system. In this paper modeling and numerical simulation of the dynamic control process is carried out to complete a comprehensive numerical simulation of an AO system.

The dynamic control process plays a very important role in the practical application of an AO system. It determines whether the AO system can work stably and it relates closely to the temporal bandwidth and the phase compensation effectiveness of the system. Very limited articles have been published on the topic of the investigation and calculation of the dynamic control process and the related performances. Moreover, most of them are based on analyses of the automatic control theory (see, for example, Refs. 5, 6). The current authors employ another approach, a pure numerical simulation, to simulate and investigate the dynamic control process in an AO system. This is a continuation and expansion of our existing work. ${ }^{3,4}$

Including the dynamic control process in a numerical simulation of an AO system can only provide the computational results of long-exposure Strehl ratio for describing the phase compensation effectiveness of the AO system and it is difficult in this way to judge the working stability of the AO system effectively. Especially, it is impossible through this way to provide the bandwidth, phase margin and gain margin which are very important parameters for experimenters. In experiment, it is common to directly measure the frequency response characteristics of an AO system by using a frequency response analyzer or a frequency spectrum analyzer to obtain a Bode plot and further to achieve the bandwidth and margins. In order to realize a complete numerical simulation of an AO system, besides including a numerical simulation of the dynamic control process, a numerical simulation of the frequency response characteristics of an AO system must be included as well. In other words, a numerical simulation of a frequency response analyzer or a frequency spectrum analyzer must be included in the AO numerical simulation. Another purpose of this paper is to carry out a numerical simulation of the frequency response characteristics in an $\mathrm{AO}$ system.

\section{NUMERICAL SIMULATION OF THE DYNAMIC CONTROL PROCESS}

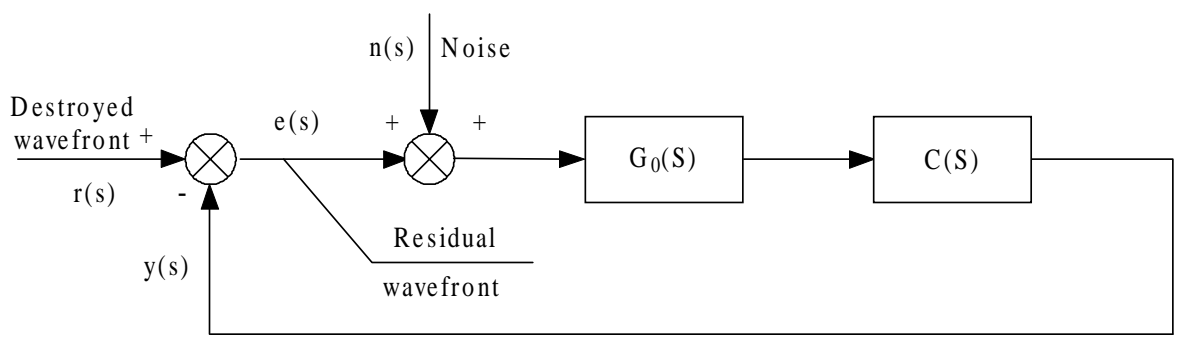

Fig.1 Block diagram of a typical adaptive optics (AO) system.

A block diagram of a typical AO system is shown in Fig. 1. In Fig. 1, $r(s)$ is the distorted wavefront of incident optical wave field, $y(s)$ is the corrected wavefront from the AO system, $e(s)$ is the residual wavefront, $n(s)$ is noise in the system, $G_{0}(s)$ is the transfer function of the $\mathrm{AO}$ system itself (without a controller), and $C(s)$ is the transfer function of a controller, where $s=j 2 \pi f$ is the Laplacian and $f$ is the frequency.

A conventional method for determining $G_{0}(s)$ is to do a theoretical analysis of a realistic AO system by utilizing the automatic control theory in combination with experiences. First, a formula of transfer function is assumed for the system 
and compared with the experimental data. After some adjustments, the most proper expression is chosen. If a proper expression for $C(s)$ can be found by utilizing a theoretical analysis of the automatic control theory at the same time, it is convenient to analyze and investigate the dynamic control performance in an AO system by utilizing the automatic control theory. Xinyang $\mathrm{Li}^{6,7}$ did careful measurements and investigations for a 61 units $\mathrm{AO}$ system ${ }^{8}$ with frame rate of $838 \mathrm{~Hz}$ and presented the transfer function of the AO system itself (without a controller)

$$
G_{0}(s)=\left(\frac{1-e^{-T s}}{T s}\right)^{2} \bullet K_{0} \bullet e^{-\tau_{0} s},
$$

where $\tau_{0}$ is the effective time delay, $K_{o}$ is the total gain of the system, and $T$ is the sampling time period. The phase response of the $\mathrm{AO}$ system itself is expressed by

$$
\phi(f)=2 \pi f\left(T+\tau_{0}\right) .
$$

These expressions have been proved to agree with the experimental data excellently. In this paper, these expressions are chosen as a comparison standard to substitute for the experimental data.

In the practical operation of an $\mathrm{AO}$ system hardware, a difference equation for the entire $\mathrm{AO}$ system (including the $\mathrm{AO}$ system itself and a controller) plays the essential role. The difference equation is

$$
U_{n}=a_{1} U_{n-1}+a_{2} U_{n-2}+a_{3} U_{n-3}+\cdots+b_{1} E_{n}+b_{2} E_{n-1}+b_{3} E_{n-2}+\cdots .
$$

This difference equation connects the corrected wavefront at the $n$th time step $U_{n}$ with the corrected wavefronts at the foregoing time steps $U_{n-1}, U_{n-2}, \ldots$ and the residual wavefronts at the present time step and the foregoing time steps $E_{n}, E_{n-1}$, $E_{n-2}, \ldots$ A different controller shows a different performance in terms of the difference of this connection, i.e. in terms of the difference of the coefficient combination in Eq. (3).

Controllers in practical use can be divided into two kinds: digital controllers and continuous controllers. For the digital controllers, their expression $C(Z)$ can be directly deduced from a theoretical analysis of the automatic control theory as

$$
C(Z)=\frac{b_{1}+b_{2} Z^{-1}+b_{3} Z^{-2}+\ldots}{1-a_{1} Z^{-1}-a_{2} Z^{-2}-a_{3} Z^{-3}-\ldots}
$$

The coefficients in Eq. (4) correspond to those in Eq. (3). However, for the continuous controllers, it is necessary to obtain their expression of transfer function $C(s)$ from a theoretical analysis of the automatic control theory first, then chose a proper transformation method to obtain the corresponding $C(Z)$. It must be noted that by using different transformation method a different discretization expression i.e. different coefficient combination can be produced. There is no one-to-one determinacy. Furthermore, in practice it is often quite difficult to obtain accurate expressions for $G_{0}(s)$ and $C(s)$, especially, in an AO system in design. Thus, the above-mentioned analytical method based on the automatic control theory has its limitation.

On the other hand, a coefficient combination may be proved to correspond to a controller with very good performances either by a numerical simulation or by the practical operation of the system hardware, but it may be difficult even impossible to find its $C(s)$ by using a theoretical analysis of the automatic control theory. That is, the preferable coefficient combination may not correspond to a controller which expression of the transfer function has been known. On the contrary, 
a numerical simulation can directly simulate the operation status of an $\mathrm{AO}$ system hardware and a simulation computation can be carried out for an arbitrary interested coefficient combination. Thus, in comparison to a theoretical analysis of the automatic control theory, a numerical simulation of the dynamic control process in an AO system is advantageous.

An AO system can be used to detect and correct all stationary wavefront distortion in the optical path from the observed object to the imaging system and the dynamic wavefront distortion come from the turbulent medium to improve the image quality. Also, an AO system can be used to detect and correct dynamic wavefront distortion of the optical wave from a beacon through the turbulent atmosphere and the wavefront of a laser beam from the outgoing lasers is phase-compensated before propagating in the turbulent atmosphere so that the beam quality on the target is significantly improved. The numerical simulation in this paper is for the second application of an $\mathrm{AO}$ system. Note that a numerical simulation presented in this paper is also suitable to the first application after small revising.

Wenhan Jiang and his colleagues in the Institute of Optics and Electronics, Chinese Academy of Sciences designed and manufactured a 61 element AO system. ${ }^{8}$ This AO system includes a Hartmann-Shack (HS) wavefront sensor with 48 subapertures. Its frame rate is $838 \mathrm{~Hz}$. The number of actuator in the deformable mirror is 61. An optical wave from a beacon propagates through a turbulent atmosphere to reach the HS sensor with a distorted wavefront. The incident optical wave is divided into several subapertures according to the practical pattern in the HS sensor. The optical wave field of each subaperture is focused at discrete CCD pixels of a certain number and the centroid displacements with respect to the centroid without wavefront distortion are obtained. The distorted wavefront is reconstructed by a reconstructor from the measured average slopes of all subapertures. The reconstructed wavefront is handled in a controller and then corrected by a deformable mirror and a 2-axis fast steering mirror. Finally, a phase-compensated beam from the outgoing lasers propagates in the same, but translated (because of the time delay in the AO system and the lateral wind and/or lateral movements of target and outgoing laser) turbulent medium again to reach the target. All the procedures described above are carried out according to the realistic working procedures and timetable of the AO system. A number of patterns at target corresponding to different time steps are accumulated to obtain a long-exposure pattern. A centroid is determined from the long-exposure pattern. The Strehl ratio $S$ of the optical energies within a circle around the centroid with a radius of the first dark ring in the Airy pattern after propagations through the turbulent medium and through a vacuum is used to evaluate the performance of an AO system. The $S$ in this paper is the STRCC in Ref. 3.

A numerical simulation of the dynamic control process in an AO system includes a series of dynamic iterations. In detail, each dynamic iteration includes the following procedures (refer to Ref. 3). First, the distorted wavefront from the beacon after propagating through the turbulent atmosphere at a certain time step is computed. Second, a simulation computation of wavefront detection and wavefront reconstruction is carried out to obtain $E_{n}$ corresponding to this time step. Third, utilizing the coefficient combination corresponding to the controller in use, a new corrected wavefront $U_{n}$ can be calculated from Eq. (3) in terms of $E_{n}$ and the foregoing $E_{n-1} \ldots, U_{n-1} \ldots$ Fourth, according to the practical working procedures and timetable, at the moment adding the time delay the phase-compensated beam from the outgoing lasers propagates in the translated turbulent medium (corresponding to translated phase screens in the numerical simulation) to reach the target. Such dynamic iteration calculation is carried out for each time step. At the next time step the dynamic iteration calculation is repeated and the only difference is that the phase screens are translated a small amount according to the timetable and the lateral speed(s). A series of dynamic iteration calculations continue to obtain the useful performance metrics such as long-exposure Strehl ratio, instantaneous Strehl ratio for each time step, two-dimensional phase and intensity distributions at target. All these 
performance metrics change with time.

If the coefficients in the controller were chosen improperly, the AO system would not work stably. In principle, this instability will appear in long time dynamic iterations. In fact, such instabilities were really observed in our numerical simulations under some conditions. However, such instabilities often appear after thousands even more dynamic iterations. Obviously, it is not effective to utilize a dynamic iteration approach to investigate the stability in an AO system. Furthermore, only by means of the dynamic iteration it is impossible to obtain the bandwidth and margins which are very important for the experimenters. This is why we must do a numerical simulation of the frequency response characteristics in an AO system in addition to a numerical simulation of the dynamic control process.

In order to directly compute the long-exposure Strehl ratio of an AO system, it is necessary to carry out a great number of dynamic iterations under a working condition. So, it is very time-consuming to choose an optimum working condition in an AO system only based on the dynamic iteration computation. We hope to find a more convenient and more effective approach to make a preliminary choice of the optimum working condition. Then, the time-consuming computation of long-exposure Strehl ratio will be only done under some chosen conditions mainly as a tool of testing and verification. This is another reason for us to carry out a numerical simulation of the frequency response characteristics in an AO system.

\section{NUMERICAL SIMULATION OF THE FREQUENCY RESPONSE CHARACTERISTICS}

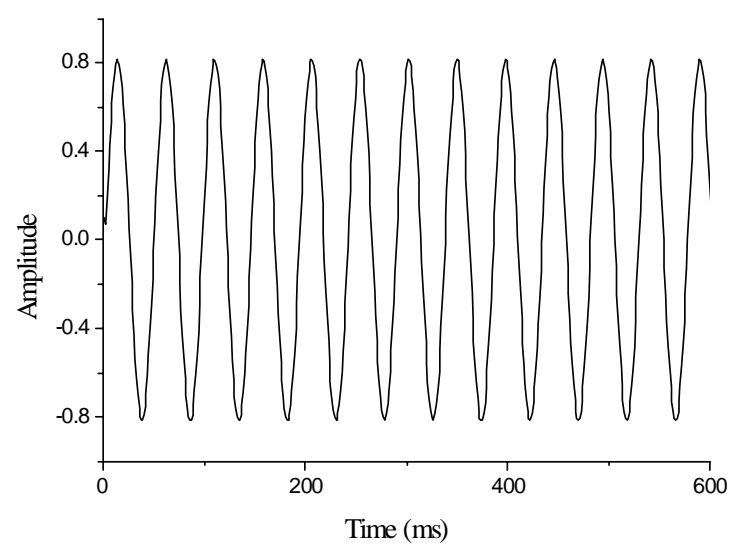

Fig. 2 Diagram of the simulated frequency response signal of an AO system itself (without controller). Input: sine-wave signal with amplitude of 1 .

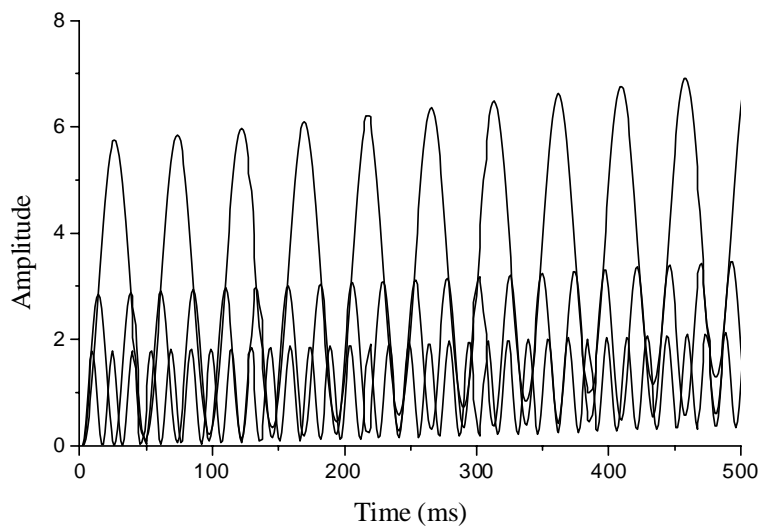

Fig. 3 Diagram of the simulated frequency response signals of an open-loop AO system with an unstable controller at frequencies of $25 \mathrm{~Hz}, 50 \mathrm{~Hz}, 80 \mathrm{~Hz}$. Input: sine-wave signals with amplitude of 1 .

To do the numerical simulation of the frequency response characteristics, a simulation calculation of a frequency response analyzer or a frequency spectrum analyzer is included in our modeling of an AO system. Similar to the operation in an experiment, a sine-wave signal with a certain amplitude (taking the input amplitude as 1) and a certain frequency is applied on an arbitrarily chosen actuator (the chosen actuator is generally located neither in the central region nor at the periphery of 
the deformable mirror) as an input signal to the entire AO system. After the treatment in the AO system, a frequency response signal which changes with time (as a sine-wave with the same frequency but different amplitude and phase) can be detected at the same actuator. Several examples of the frequency responses are shown in Figs. 2 and 3. Then, a Fourier analysis is applied to the frequency response signal to obtain the amplitude response and phase response of the AO system with a controller or without a controller. Such simulation computational results for a number of different frequencies form a Bode plot of the system. Further, useful parameters such as bandwidth, phase margin and gain margin can be obtained from the Bode plot.

The inputted sine-wave signal can be of a single frequency and such computation is called as a single-frequency simulation computation. This mode corresponds to the experimental operation of a frequency response analyzer. The inputted sine-wave signal can be of multiple frequencies also and such computation is called as a multi-frequency simulation computation. This mode corresponds to the experimental operation of a frequency spectrum analyzer. Apparently, frequency responses at many frequencies can be obtained in one time of the multi-frequency simulation computation. Therefore, its computing efficiency is much higher than that of the single-frequency computation.

When a number of sine-wave components with different frequencies are combined into a composite input signal, the initial amplitude of each component can be different. A component with different initial amplitude in the composite input may produce a different simulation computational result of the frequency response. In the simulation computation by using a multi-frequency input, the initial amplitude of a composite input signal after composing together can be divided by its maximum amplitude, then the resultant composite signal is used as the input signal of the AO system to calculate the frequency response. This process is called as normalization of the initial amplitude. The computational results presented in the next section show that the normalization of the initial amplitude has a great influence on the simulation computational results of the frequency response.

\section{RESULTS AND DISCUSSIONS}

\subsection{Frequency response signal}

When a sine-wave of single frequency is applied on the AO system substituting for the distorted wavefront $r(s)$ and the AO system is in the open-loop state, the output signal of the system frequency response of a certain frequency is shown in Fig. 2. The signal is a sine-wave with the same frequency but different amplitude and phase in comparison to the input signal.

It is found that when the AO system includes a controller the slope of frequency response signal is closely related to the stability of the whole system. According to the automatic control theory, when the coefficient in Eq. (3) $a_{l}>1$ the system is instable. The open-loop frequency response signals of different frequencies in an AO system with a controller which coefficients are $a_{1}=1.001, b_{1}=0.55$, others $=0$ are shown in Fig. 3. It can be seen from Fig. 3 that the evolutions of the frequency response signals with time significantly slope upwards. On the contrary, the instability under the same condition can not be found in the long-exposure Strehl ratio computation even after carrying out thousands dynamic iterations. On the other hand, under a large number of conditions of $a_{l}=1.00$ and $a_{1}<1.00$ the corresponding computational results of the evolution of the frequency response signal with time show to be horizontal or slope downwards. In conclusion, it is a very effective tool to observe whether the evolution of the frequency response signal with time slopes upwards or not and if 
Table 1 Numerical simulation results of frequency response of a 61 element AO system with frame rate of $838 \mathrm{~Hz}$ by using single frequency and multiple frequency inputs

\begin{tabular}{|c|c|c|c|c|c|c|c|c|}
\hline \multirow[t]{2}{*}{$\begin{array}{l}\text { Frequency } \\
\qquad(\mathrm{Hz})\end{array}$} & \multicolumn{2}{|c|}{$\begin{array}{l}\text { Best fit of the } \\
\text { experimental data }\end{array}$} & \multicolumn{2}{|c|}{$\begin{array}{l}\text { Numerical simulation } \\
\text { results by using } \\
\text { single frequency } \\
\text { input }\end{array}$} & \multicolumn{2}{|c|}{$\begin{array}{l}\text { Numerical simulation } \\
\text { results by using composite } \\
\text { input of } 16 \text { frequencies } \\
\text { with initial amplitudes of } \\
1 \text { and normalization }\end{array}$} & \multicolumn{2}{|c|}{$\begin{array}{l}\text { Numerical simulation results } \\
\text { by using composite input of } \\
500 \text { frequencies with initial } \\
\text { amplitudes of } 1 \text { and } \\
\text { normalization } \\
\end{array}$} \\
\hline & $\operatorname{Gain}(\mathrm{dB})$ & Phase $\left(^{\circ}\right)$ & $\operatorname{Gain}(\mathrm{dB})$ & Phase $\left(^{\circ}\right)$ & Gain(dB) & Phase $\left(^{\circ}\right)$ & $\operatorname{Gain}(\mathrm{dB})$ & Phase $\left(^{\circ}\right)$ \\
\hline 20.85 & -1.78 & -23.26 & -1.78 & -23.34 & -1.82 & -23.66 & -1.71 & -21.16 \\
\hline 29.20 & -1.79 & -32.59 & -1.80 & -32.67 & -1.84 & -33.14 & -1.70 & -30.65 \\
\hline 41.71 & -1.83 & -46.54 & -1.84 & -46.67 & -1.87 & -47.36 & -1.70 & -44.87 \\
\hline 54.22 & -1.88 & -60.49 & -1.89 & -60.67 & -1.92 & -61.59 & -1.72 & -59.09 \\
\hline 66.73 & -1.94 & -74.47 & -1.95 & -74.67 & -1.99 & -75.82 & -1.76 & -73.31 \\
\hline 79.25 & -2.02 & -88.43 & -2.03 & -88.67 & -2.07 & -90.04 & -1.81 & -87.52 \\
\hline 100.10 & -2.18 & -111.71 & -2.19 & -112.01 & -2.22 & -113.75 & -1.94 & -111.19 \\
\hline 120.95 & -2.37 & -135.00 & -2.38 & -135.35 & -2.42 & -137.45 & -2.12 & -134.85 \\
\hline 141.81 & -2.60 & -158.25 & -2.62 & -158.68 & -2.65 & -161.17 & -2.34 & -158.50 \\
\hline 166.83 & -2.92 & -173.80 & -2.95 & 173.32 & -2.98 & 170.38 & -2.67 & 173.14 \\
\hline 196.03 & -3.37 & 141.26 & -3.41 & 140.65 & -3.44 & 137.19 & -3.13 & 140.08 \\
\hline 225.23 & -3.90 & 108.64 & -3.95 & 107.98 & -3.98 & 104.01 & -3.68 & 107.05 \\
\hline 262.76 & -4.70 & 66.75 & -4.77 & 65.98 & -4.79 & 61.34 & -4.53 & 64.63 \\
\hline 308.64 & -5.88 & 15.50 & -5.97 & 14.65 & -5.98 & 9.19 & -5.78 & 12.85 \\
\hline 358.69 & -7.42 & -40.30 & -7.55 & -41.35 & -7.55 & -47.70 & -7.41 & -43.59 \\
\hline 415.42 & -9.55 & -103.65 & -9.62 & -105.00 & -9.59 & -112.36 & -9.14 & -109.96 \\
\hline
\end{tabular}

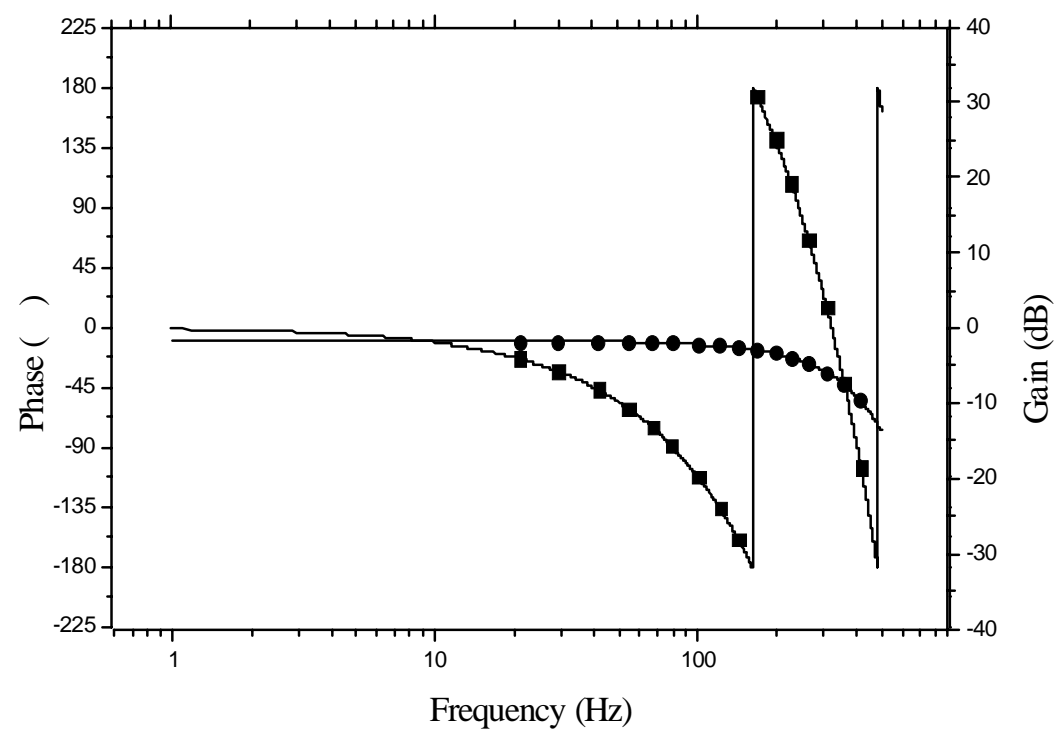

Fig. 4 Bode plot of an AO system itself (without a controller).

Wherein solid lines represent the best fits of experimental data, dot represents the numerical simulation results of amplitude response, and square represents the numerical simulation results of phase response. 
the evolution slopes upwards the system is instable. This seems a much more effective tool for judging the instability in an AO system than a dynamic iteration computation of the long -exposure Strehl ratio.

\subsection{Computation of frequency response characteristics by using a single frequency input}

Numerical simulation results of the frequency response in a realistic AO system ${ }^{8}$ itself (without a controller) by using a single frequency input are shown in Table 1. From our analyses and the results in Table 1, it can be deduced that in our simulation computation system $K_{o}$ in Eq. (1) is approximately equal to $-1.78 d B$ and $T+\tau_{0}$ in Eq. (2) is chosen as 3.07 microsecond. After substituting these values into Eqs. (1) and (2), the corresponding Bode plot can be drawn and it is shown in Fig. 4. In Fig. 4, the solid lines are the results from Eqs. (1) and (2) and as stated above they can be seen as the best fits of experimental data. In addition, symbols in Fig. 4 are our numerical simulation results by using a single frequency input. It can be seen that the agreement of our simulation computational results with the experimental data is excellent. This excellent agreement shows our simulation computation of the frequency response in an AO system is reliable. The numerical comparison is shown in Table 1.

It is found from our simulation computation of the frequency response that there are two integration elements in the AO system. They correspond to the sampling process in the HS wavefront sensor and the response process of the actuator in the deformable mirror, respectively.

\subsection{Computation of frequency response characteristics by using a multi-frequency input}

Table 2 Numerical simulation results of frequency response of a 61 element AO system with frame rate of $838 \mathrm{~Hz}$ by using 16 frequencies composite inputs

\begin{tabular}{|c|c|c|c|c|c|c|c|c|c|c|c|c|}
\hline \multirow[t]{2}{*}{$\begin{array}{l}\text { Frequ- } \\
\text { ency } \\
(\mathrm{Hz})\end{array}$} & \multicolumn{2}{|c|}{$\begin{array}{c}\text { All initial } \\
\text { amplitudes of } 1\end{array}$} & \multicolumn{2}{|c|}{$\begin{array}{l}\text { Initial amplitudes } \\
\text { proportional to } \\
\text { the frequencies }\end{array}$} & \multicolumn{2}{|c|}{$\begin{array}{l}\text { Initial amplitudes } \\
\text { inversely } \\
\text { proportional to } \\
\text { the frequencies }\end{array}$} & \multicolumn{2}{|c|}{$\begin{array}{c}\text { All initial } \\
\text { amplitudes of } 1 \\
\text { with } \\
\text { normalization }\end{array}$} & \multicolumn{2}{|c|}{$\begin{array}{l}\text { Initial amplitudes } \\
\text { proportional to } \\
\text { the frequencies } \\
\text { with } \\
\text { normalization }\end{array}$} & \multicolumn{2}{|c|}{$\begin{array}{l}\text { Initial amplitudes } \\
\text { inversely propor- } \\
\text { tional to the } \\
\text { frequencies with } \\
\text { normalization }\end{array}$} \\
\hline & $\begin{array}{l}\text { Gain } \\
(\mathrm{dB})\end{array}$ & $\begin{array}{c}\text { Phase } \\
\left({ }^{\circ}\right) \\
\end{array}$ & $\begin{array}{l}\text { Gain } \\
(\mathrm{dB})\end{array}$ & $\begin{array}{c}\text { Phase } \\
\left({ }^{\circ}\right) \\
\end{array}$ & $\begin{array}{l}\text { Gain } \\
(\mathrm{dB})\end{array}$ & $\begin{array}{c}\text { Phase } \\
\left({ }^{\circ}\right)\end{array}$ & $\begin{array}{l}\text { Gain } \\
(\mathrm{dB})\end{array}$ & $\begin{array}{c}\text { Phase } \\
\left({ }^{\circ}\right)\end{array}$ & $\begin{array}{l}\text { Gain } \\
(\mathrm{dB})\end{array}$ & $\begin{array}{c}\text { Phase } \\
\left({ }^{\circ}\right) \\
\end{array}$ & $\begin{array}{l}\text { Gain } \\
(\mathrm{dB})\end{array}$ & $\begin{array}{l}\text { Phase } \\
\left({ }^{\circ}\right)\end{array}$ \\
\hline 20.85 & -1.00 & -23.60 & -58.74 & 117.91 & -184 & -23.70 & -1.82 & -23.66 & -1.82 & -23.03 & & -23.71 \\
\hline 29.20 & -0.97 & -33.13 & -56.23 & 7.43 & -1.86 & -33.18 & -1.84 & -33.14 & -1.82 & -32.75 & -1. & -33.18 \\
\hline 41.71 & -0.92 & -47.35 & -55.46 & 05.84 & -1.90 & -47.40 & -1.87 & & -1.87 & -47.07 & -1. & -47.39 \\
\hline 54.22 & -0.85 & & -63.8 & -50.86 & -1.95 & -61.62 & -1.92 & -61.59 & -1.90 & -01.59 & -1.8 & -61.63 \\
\hline & & & & & & & & & & & & 5.85 \\
\hline 79.25 & 100 & & -66.93 & & & -90.07 & -2.07 & & -2.05 & -89.91 & & -90.09 \\
\hline 100.10 & -1.22 & -113.73 & -54.64 & -111.13 & -2.25 & -113.76 & -2.22 & -113.75 & -2.20 & -113.67 & -2.2 & -113.77 \\
\hline 120.95 & -1.47 & -137.50 & -74.27 & 57.74 & -2.45 & -137.46 & -2.42 & -137.45 & -2.40 & -137.40 & -2.40 & -137.43 \\
\hline 141.81 & & & & & & & & & & & & \\
\hline 166.83 & & & -60.07 & & & & & & -2.97 & 170.40 & -2.97 & 170.40 \\
\hline 196.03 & 256 & & -68.78 & & & & -3.44 & & -3.43 & & -3.45 & 137.24 \\
\hline 225.23 & -3.19 & 103.99 & -66.55 & 107.71 & -4.01 & 104.06 & -3.98 & 104.01 & -3.97 & 104.00 & -3.98 & 104.08 \\
\hline 262.76 & -3.91 & & & & & & & & & & & 61.44 \\
\hline 308.64 & -5.09 & & -69.51 & & -6.01 & 9.32 & -5.98 & 9.19 & -5.97 & 9.15 & -5.99 & 9.33 \\
\hline 358.69 & -6.80 & -47.69 & -72.68 & -53.68 & -7.57 & -47.50 & -7.55 & -47.7 & -7.54 & -47.76 & -7.56 & -47.50 \\
\hline 415.42 & -8.67 & -112.35 & -73.91 & -117.94 & -9.60 & -112.04 & -9.59 & -112.36 & -9.59 & -112.43 & -9.56 & -112.05 \\
\hline
\end{tabular}


Corresponding to the working status of the frequency spectrum analyzer, a composite input signal of tens even hundreds different frequencies can be used in our numerical simulation as the driving signal. After a large number of numerical experiments it is found that reasonable and more accurate numerical simulation results by using multi-frequency inputs can be obtained only after normalizing the composite initial amplitude, whereas it seems not very important whether the initial amplitudes of components with different frequencies are equal to each other or not. The initial amplitude of each component corresponds to the weight of the contribution of that component. Numerical simulation results by using a composite input of 16 frequencies with different initial amplitudes and with or without normalization of the initial amplitude are shown in Table 2. Composite inputs of multiple-frequency with initial amplitude of 1 and normalization are used in our numerical simulation computation. Numerical simulation results by using composite inputs of 16 and 500 frequencies are shown in Table 1 also for comparing with the single-frequency results and the experimental data.

Results in Table 1 show that the multi-frequency results are good enough for the purpose of obtaining the interesting bandwidth and margins although the multi-frequency results have small differences in comparison with the single-frequency results in the lower frequency region and the amplitude responses of the multi-frequency results do not completely monotonically decrease in some region. When the multi-frequency results are presented in Fig. 4, the small differences between the multi-frequency results and the single-frequency results are not distinguishable although these small differences can be seen from values in Table 1. It means that the multi-frequency results of a numerical simulation agree with the experimental data as excellently as the single-frequency results do and they are good enough for drawing a Bode plot and calculating the bandwidth and margins. However, the computing time by using a multi-frequency input will be much less than that by using single-frequency inputs. It is also shown that the components with different frequencies may have some interaction. It means that the measurement by using a frequency spectrum analyzer possibly includes some error in comparison to the measurement by using a frequency response analyzer.

\subsection{Results of an AO system with a PI (Proportional plus Integral) controller}

In fact, an AO system in practical use has a controller for controlling the dynamic process in the system. The PI (Proportional plus Integral) controller is a fundamental controller and is widely used in an AO system. This is why this kind of controller is chosen in our numerical simulation. Apparently, any other kind of controller of an AO system can be used in our numerical simulation in a similar way. For the PI controller, $C(s)=K / s$ where $K$ is a constant and it is the total gain of the system. Its corresponding difference equation is Eq. (3) with $a_{l}=1.0, b_{l}=K T$ where $T$ is the sampling time period.

The important parameters interested by experimenters such as bandwidth, phase margin and gain margin can be obtained from our numerical simulation of the frequency response characteristics in an AO system with a PI controller under the conditions of different $K$ and the corresponding phase compensation effectiveness can be evaluated by the long-exposure Strehl ratios under different experimental conditions which can be computed by our numerical simulation of the dynamic control process of the AO system. These computational results are shown in Table 3. It can be seen that under different experimental conditions the coefficient combination of a controller corresponding to the optimum phase compensation effectiveness is different; and there is no simple relation between the optimum phase compensation effectiveness and the largest bandwidth in an AO system. It appears that in order to do a complete evaluation of performances of an AO system, the bandwidth and margins of the controller and the optimum phase compensation effectiveness of the AO system must be combined together with some analyses and experiences. Work in this aspect is in progress. 
Table 3 Numerical simulation results of bandwidth, phase margin, gain margin and Strehl ratio in an AO system with a PI controller

Computational conditions: The 61 element $\mathrm{AO}$ system with frame rate of $838 \mathrm{~Hz}, 1000$ times of dynamic iteration, propagation distance of 3 kilometers, $C_{n}^{2}=10^{-14} \mathrm{~m}^{-2 / 3}$, the lateral distance between target and beacon $X_{0}=0, V_{c}$ is the velocity of target, $V_{0}$ is the lateral wind speed. Condition 1: $V_{0}=5 \mathrm{~m} / \mathrm{s}, V_{c}=0$; Condition $2: V_{0}=10 \mathrm{~m} / \mathrm{s}, V_{c}=0$; Condition 3: $V_{0}=5 \mathrm{~m} / \mathrm{s}, V_{c}=-50 \mathrm{~m} / \mathrm{s}$.

\begin{tabular}{|c|c|c|c|c|c|c|c|}
\hline$a_{I}$ & $b_{l}$ & $\begin{array}{c}\text { Bandwidth } \\
(\mathrm{Hz})\end{array}$ & $\begin{array}{c}\text { Phase } \\
\text { margin }\left({ }^{\circ}\right)\end{array}$ & $\begin{array}{c}\text { Gain } \\
\text { margin } \\
(\mathrm{dB})\end{array}$ & $\begin{array}{c}\text { Strehl ratio } \\
\text { Condition 1 }\end{array}$ & $\begin{array}{c}\text { Strehl ratio } \\
\text { Condition 2 }\end{array}$ & $\begin{array}{c}\text { Strehl ratio } \\
\text { Condition 3 }\end{array}$ \\
\hline 1.00 & 0.20 & 21.7 & 70.1 & 13.5 & 0.171 & 0.072 & 0.093 \\
\hline 1.00 & 0.25 & 26.7 & 64.7 & 11.6 & 0.204 & 0.078 & 0.092 \\
\hline 1.00 & 0.30 & 32.5 & 60.1 & 10.0 & 0.233 & 0.082 & 0.090 \\
\hline 1.00 & 0.35 & 37.5 & 55.5 & 8.6 & 0.257 & 0.084 & 0.087 \\
\hline 1.00 & 0.40 & 43.4 & 50.2 & 7.4 & 0.274 & 0.084 & 0.083 \\
\hline 1.00 & 0.45 & 48.4 & 45.6 & 6.4 & 0.285 & 0.082 & 0.077 \\
\hline 1.00 & 0.50 & 53.5 & 40.2 & 5.5 & 0.286 & 0.078 & 0.071 \\
\hline 1.00 & 0.55 & 58.9 & 35.0 & 4.7 & 0.278 & 0.072 & 0.062 \\
\hline 1.00 & 0.60 & 64.2 & 31.2 & 3.9 & 0.260 & 0.063 & 0.053 \\
\hline
\end{tabular}

\section{CONCLUSIONS}

In this paper, modeling and a numerical simulation of the dynamic control process and the frequency response characteristics in an AO system are carried out, respectively and combined for evaluating performances of the AO system. Numerical simulation investigations show that (i) the long-exposure Strehl ratio and other useful metrics under different experimental conditions can be computed by a numerical simulation of the dynamic control process in the AO system. (ii) Important parameters interested by experimenters such as bandwidth, phase margin and gain margin can be obtained from a numerical simulation of the frequency response characteristics in an AO system. (iii) Our simulation computational results of the frequency response characteristics in an AO system agree with the experimental data excellently. (iv) Both the numerical simulation results by usfing single-frequency inputs and those by using multi-frequency inputs can be used to obtain the bandwidth and margins in an AO system. However, the computing efficiency by using multi-frequency inputs is much higher than that by using single-frequency inputs. (v) In order to get an insight investigation and a complete evaluation of performances in an AO system, a numerical simulation of the dynamic control process of the AO system and a numerical simulation of the frequency response characteristics must be combined. It is shown that a numerical simulation is very valuable and helpful in design, examination, investigation and application of a highly complex electro-optic system such as an AO system.

\section{ACKNOWLEDGEMENT}

The authors gratefully acknowledge the many helpful discussions with Dr. Xinyang Li of the Institute of Optics and Electronics, Chinese Academy of Sciences. 


\section{REFERENCES}

1. R.K.Tyson, Principles of Adaptive Optics, 2nd Ed., Academic Press, Boston, 1997.

2. M.C.Roggeman, B.Welsh, Imaging Through Turbulence, CRC Press, Boca Raton, Florida, 1996.

3. Hai-Xing Yan, Shu-Shan Li, De-Liang Zhang, She Chen, "Numerical simulation of an adaptive optics system with laser propagation in the atmosphere," Appl. Opt. 39, pp. 3023-3031, 2000.

4. Hai-Xing Yan, She Chen, Shu-Shan Li, "Numerical simulation investigations of the effects of noise and detection error in an adaptive optics system," Proc. SPIE 4494, a companion paper, 2001.

5. M.Demerle, P.Y.Madec, G.Rousset, "Servo-loop analysis for adaptive optics", in Adaptive Optics for Astronomy (eds. D.M.Alloin and J.-M.Mariotti) pp.73-88, Kluwer Academic Publishers, Netherlands, 1994.

6. Xinyang Li, Wenhan Jiang, "Control bandwidth analysis of adaptive optical systems," Proc. SPIE 3126, pp. 447-454, 1997.

7. Xinyang Li, "Control algorithm design and control performance analysis of an adaptive optics system," Thesis of the Institute of Optics and Electronics, Chinese Academy of Sciences, 1997. (in Chinese)

8. Wenhan Jiang, Chunhong Wang, Ning Ling, Xubin Wu, Hao Xian, Xinyang Li, Chunlin Guan, Mei Li, Zhiben Gong, Yi Wu, Yingjian Wang, "61 element adaptive optics system," Chinese Journal of Quantum Electronics 15, pp.193-199, 1998. (in Chinese) 